\title{
Cryptanalysis of MD4
}

\author{
Hans Dobbertin \\ German Information Security Agency \\ P. O. Box 200363 \\ D-53133 Bonn \\ e-mail: dobbertineskom.rhein.de
}

\begin{abstract}
In 1990 Rivest introduced the hash function MD4. Two years later RIPEMD, a European proposal, was designed as a stronger mode of MD4. Recently we have found an attack against two of three rounds of RIPEMD. As we shall show in the present note, the methods developed to attack RIPEMD can be modified and supplemented such that it is possible to break the full MD4, while previously only partial attacks were known. An implementation of our attack allows to find collisions for MD4 in a few seconds on a PC. An example of a collision is given demonstrating that our attack is of practical relevance.
\end{abstract}

\section{Introduction}

Rivest [7] introduced the hash function MD4 in 1990. The MD4 algorithm is defined as an iterative application of a three-round compress function. After an unpublished attack on the first two rounds of MD4 due to Merkle and an attack against the last two rounds by den Boer and Bosselaers [2], Rivest introduced the strengthened version MD5 [8]. The most important difference to MD4 is the adding of a fourth round.

On the other hand the stronger mode RIPEMD [1] of MD4 was designed as a European proposal in 1992. The compress function of RIPEMD consists of two parallel lines of a modified version of the MD4 compress function. In [4] we have shown that if the first or the last round of its compress function is omitted, then RIPEMD is not collision-free.

Recently Vaudenay [9] described another attack against the first two rounds of MD4. The two round collisions he found form almost-collisions for the full MD4. But none of the previously known partial attacks can be generalized to the three-round MD4. Thus, although MD4 has generally been considered to be weak, the original conjecture that MD4 is collision-free has still remained to be disproved. This will be done in the present paper.

We shall show that the methods developed to attack RIPEMD can be applied to MD4 very effectively. We shall derive an algorithm which allows to compute collisions for the full MD4 in a few seconds on a PC with Pentium processor. Finally it is demonstrated that a further development of our attack allows to find collisions for meaningful messages. Therefore we suggest that MD4 should no longer be applied in practice. 
It remains a challenge trying to attack MD5 with the techniques presented in this note.

\section{Terminology and basic notations}

Using the term "collision of a compress function" we assume that the corresponding initial values coincide for both inputs. For "pseudo-collisions" this is not required. But the latter are of much less practical importance and will not be considered here.

Throughout, all occuring variables and constants are 32-bit quantities. Accordingly the value of an expression is its remainder modulo $2^{32}$, and equations are to be understood modulo $2^{32}$. The symbols $\wedge, \vee, \oplus$ and $\neg$ are used for bitwise AND, OR, XOR, and complement, respectively. For a 32-bit word $W$, let $W \ll$ denote the 32 -bit value obtained by circularly shifting (rotation) $W$ left by $s$ bit positions for $0 \leq s<32$. If $W$ is an expression then, of course, evaluate it before shifting. Further we agree that $-W^{\ll s}$ stands for $-\left(W^{\ll}\right)$.

A definition of the compress function of MD4 can be found in the Appendix.

\section{Main Result and Plan of the Attack}

The main result of this paper is:

$M D_{4}$ is not collision-free.

There is an algorithm such that the finding of collisions for MD4 requires the same computational effort as about $2^{20}$ computations of the MD4-compress function.

The most direct way trying to get a collision for an iterated hash function like MD4 is trying to find a collision for the compress function with the fixed initial value required at the beginning of the computation of hash values. This is precisely what will be done in the present paper for MD4.

Throughout this note $X=\left(X_{i}\right)_{i<16}$ denotes a collection of 16 words, and the collection $\tilde{X}=\left(\tilde{X}_{i}\right)_{i<16}$ is defined by setting

$$
\begin{aligned}
\tilde{X}_{i} & =X_{i} \text { for }(i \neq 12), \\
\tilde{X}_{12} & =X_{12}+1 .
\end{aligned}
$$

In the sequel we demonstrate how to choose $X$ such that its MD4 hash value coincides with that of $\tilde{X}$, i.e.

$$
\text { compress }\left(I V_{0} ; X\right)=\operatorname{compress}\left(I V_{0} ; \tilde{X}\right)
$$

As in [4] the basic idea is that a (small) difference between only one of the input variables can be controlled in a way that the differences occuring in the computations of the two associated hash values are compensated at the end. 
Our attack is separated into three parts. Each part considers a certain segment of the compress function. For $n<m<48$ we therefore introduce the notation

$$
\text { compress }_{m}^{n}\left((A, B, C, D) ; X_{\varphi(n)}, \ldots, X_{\varphi(m)}\right)=\left(A^{\prime}, B^{\prime}, C^{\prime}, D^{\prime}\right)
$$

for the segment of compress from step $n$ to step $m$, where the mapping $\varphi$ is defined such that $X_{\varphi(i)}$ is applied the $i$-th step of compress. This means that the computation of compress ${ }_{m}^{n}$ starts with the "initial value" $(A, B, C, D)$, then steps $n$ to $m$ are applied with the corresponding input words $X_{\varphi(n)}, \ldots, X_{\varphi(m)}$, and the output $\left(A^{\prime}, B^{\prime}, C^{\prime}, D^{\prime}\right)$ of compress $s_{m}^{n}$ is the contents of the registers after step $m$.

Sometimes, in the above notation, we write simply $X$ instead of $X_{\varphi(n)}, \ldots$, $X_{\varphi(m)}$. (But thereby we do not necessarily assume that all $X_{i}$ are actually defined.) We have to introduce another basic notation which will be used in the sequel. For $n \leq i \leq m$ let

$$
\left(A_{i}, B_{i}, C_{i}, D_{i}\right), \text { resp. }\left(\tilde{A}_{i}, \tilde{B}_{i}, \tilde{C}_{i}, \tilde{D}_{i}\right)
$$

be the contents of the registers after step $i$ has been applied computing compress ${ }_{m}^{n}$ for the input $X$, resp. $\tilde{X}$. Further we set

$$
\Delta_{i}=\left(A_{i}-\tilde{A}_{i}, B_{i}-\tilde{B}_{i}, C_{i}-\tilde{C}_{i}, D_{i}-\tilde{D}_{i}\right) .
$$

Note that in each step only one of the registers is changed and that for instance $A_{4 k}=A_{4 k+1}=A_{4 k+2}=A_{4 k+3}(k=0,1, \ldots, 11)$.

\section{Part I: Inner almost-collisions (step 12 to step 19)}

For the attack against two-round RIPEMD [4], $X_{13}$ was the selected variable instead of $X_{12}$ and the first part was to find "inner collisions", i.e. an initial value and inputs for (both lines of) compress ${ }_{18}^{13}$ such that $\Delta_{18}=0$. Since $X_{13}$ occurs precisely in step 13 and step 18 of two-round RIPEMD-compress, this was a suitable approach to find collisions for two rounds. The main problem in the case of RIPEMD has been to handle its two parallel lines simultaneously.

However, here we deal with the three rounds of MD4. $X_{12}$ appears in each round exactly once, namely in steps 12,19 and $35 . X$ and $\tilde{X}$ give a collision if (and only if) $\Delta_{35}=0$, because $X_{12}$ appears in step 35 the last time. To achieve this we require a certain well-chosen value for $\Delta_{19}$, namely

$$
\Delta_{19}=\left(0,1^{\ll 25},-1^{\ll 5}, 0\right) .
$$

This means that the outputs of compress $\frac{12}{12}$ for $X$ and $\tilde{X}$ are close but not equal. We are not looking for inner collisions, but for inner "almost"-collisions with precisely the output difference specified above. 


\section{Part II: Differential attack modulo $2^{32}$ (step 20 to step 35)}

The value for $\Delta_{19}$ of Part I has been carefully selected such that, with a relatively high probability, this difference inherits to step 35 in a way that it is compensated by the difference between the inputs for this step, i.e. $X_{12}$ and $X_{12}+1$. (It should be emphasized that here the considered differences are not meant with respect to XOR but modulo $2^{32}$.) Based on Part I we can therefore find collisions for the compress function of MD4.

\section{Part III: Right initial value (step 0 to step 11)}

In the derived algorithm for finding collisions for the compress function there are still many variables free. Therefore it is very easy to get even collisions with an arbitrary prescribed initial value.

The next sections contain a detailed description of the three parts of our attack.

\section{Inner Almost-Collisions (Step 12 to Step 19)}

In this section we consider compress 12 , i.e. the steps $12-19$ of compress. Let $(A, B, C, D)$ be the initial value of compress 12 . Recall that $\left(A_{19}, B_{19}, C_{19}, D_{19}\right)$ denotes the output of compress 12 , i.e. the contents of the registers after step 19 for the inputs $X_{12}, X_{13}, X_{14}, X_{15}, X_{0}, X_{4}, X_{8}, X_{12}$, resp. $\left(\tilde{A}_{19}, \tilde{B}_{19}, \tilde{C}_{19}, \tilde{D}_{19}\right)$ for the inputs $X_{12}+1, X_{13}, X_{14}, X_{15}, X_{0}, X_{4}, X_{8}, X_{12}+1$.

We want to find an inner almost-collision, that are explicit values for $A, B$, $C, D$ and $X_{12}, X_{13}, X_{14}, X_{15}, X_{0}, X_{4}, X_{8}$ such that

$$
\Delta_{19}=\left(0,1^{\ll 25},-1^{\ll 5}, 0\right) \text {. }
$$

The reason for this requirement will become clear in the next section. The following table shows the contents of the registers after the application of the steps $i=12, \ldots, 19$ for $X_{12}, X_{13}, \ldots$ and for $X_{12}+1, X_{13}, \ldots$, respectively. To simplify the notations we set $A_{*}=A_{19}, B_{*}=B_{19}, \ldots, U=A_{12}, V=D_{13}, W=C_{14}$, $Z=B_{15}$ and $\tilde{U}=\tilde{A}_{12}, \tilde{V}=\tilde{D}_{13}, \tilde{W}=\tilde{C}_{14}, \tilde{Z}=\tilde{B}_{15}$.

\begin{tabular}{|c||c|c|c|c||c|c|c|c|}
\hline step & $A$ & $B$ & $C$ & $D$ & input & shift & function & constant \\
\hline \hline 12 & $U$ & $B$ & $C$ & $D$ & $X_{12}$ & 3 & $F$ & 0 \\
\hline 13 & $U$ & $B$ & $C$ & $V$ & $X_{13}$ & 7 & $F$ & 0 \\
\hline 14 & $U$ & $B$ & $W$ & $V$ & $X_{14}$ & 11 & $F$ & 0 \\
\hline 15 & $U$ & $Z$ & $W$ & $V$ & $X_{15}$ & 19 & $F$ & 0 \\
\hline 16 & $A_{*}$ & $Z$ & $W$ & $V$ & $X_{0}$ & 3 & $G$ & $K_{1}$ \\
\hline 17 & $A_{*}$ & $Z$ & $W$ & $D_{*}$ & $X_{4}$ & 5 & $G$ & $K_{1}$ \\
\hline 18 & $A_{*}$ & $Z$ & $C_{*}$ & $D_{*}$ & $X_{8}$ & 9 & $G$ & $K_{1}$ \\
\hline 19 & $A_{*}$ & $B_{*}$ & $C_{*}$ & $D_{*}$ & $X_{12}$ & 13 & $G$ & $K_{1}$ \\
\hline
\end{tabular}




\begin{tabular}{|c||c|c|c|c||c|c|c|c|}
\hline step & $A$ & $B$ & $C$ & $D$ & input & shift & function & constant \\
\hline \hline 12 & $\tilde{U}$ & $B$ & $C$ & $D$ & $X_{12}+1$ & 3 & $F$ & 0 \\
\hline 13 & $\tilde{U}$ & $B$ & $C$ & $\tilde{V}$ & $X_{13}$ & 7 & $F$ & 0 \\
\hline 14 & $\tilde{U}$ & $B$ & $\tilde{W}$ & $\tilde{V}$ & $X_{14}$ & 11 & $F$ & 0 \\
\hline 15 & $\tilde{U}$ & $\tilde{Z}$ & $\tilde{W}$ & $\tilde{V}$ & $X_{15}$ & 19 & $F$ & 0 \\
\hline 16 & $A_{*}$ & $\tilde{Z}$ & $\tilde{W}$ & $\tilde{V}$ & $X_{0}$ & 3 & $G$ & $K_{1}$ \\
\hline 17 & $A_{*}$ & $\tilde{Z}$ & $\tilde{W}$ & $D_{*}$ & $X_{4}$ & 5 & $G$ & $K_{1}$ \\
\hline 18 & $A_{*}$ & $\tilde{Z}$ & $\tilde{C}_{*}$ & $D_{*}$ & $X_{8}$ & 9 & $G$ & $K_{1}$ \\
\hline 19 & $A_{*}$ & $\tilde{B}_{*}$ & $\tilde{C}_{*}$ & $D_{*}$ & $X_{12}+1$ & 13 & $G$ & $K_{1}$ \\
\hline
\end{tabular}

Here we require $\tilde{B}_{*}+1 \ll 25=B_{*}$ and $C_{*}+1 \ll 5=\tilde{C}_{*}$. The framed entries are those which have been modified in the particular steps, and the Boolean functions $F$ and $G$ are "selection" and "majority":

$$
\begin{aligned}
& F(U, V, W)=(U \wedge V) \vee(\neg U \wedge W), \\
& G(U, V, W)=(U \wedge V) \vee(U \wedge W) \vee(V \wedge W),
\end{aligned}
$$

and $K_{1}=0 x 5 a 827999$ (see Appendix). The finding of an inner almost-collision is equivalent to the finding of a collection of solutions $B, C, A_{*}, B_{*}, C_{*}, D_{*}, U$, $V, W, Z, \tilde{U}, \tilde{V}, \tilde{W}, \tilde{Z}$ for the following system of equations:

$$
\begin{aligned}
1 & =\tilde{U}^{\ll 29}-U^{\ll 29}, \\
F(\tilde{U}, B, C)-F(U, B, C) & =\tilde{V}^{\ll 25}-V^{\ll 25}, \\
F(\tilde{V}, \tilde{U}, B)-F(V, U, B) & =\tilde{W}^{\ll 21}-W^{\ll 21}, \\
F(\tilde{W}, \tilde{V}, \tilde{U})-F(W, V, U) & =\tilde{Z}^{\ll 13}-Z^{\ll 13}, \\
G(\tilde{Z}, \tilde{W}, \tilde{V})-G(Z, W, V) & =U-\tilde{U}, \\
G\left(A_{*}, \tilde{Z}, \tilde{W}\right)-G\left(A_{*}, Z, W\right) & =V-\tilde{V}, \\
G\left(D_{*}, A_{*}, \tilde{Z}\right)-G\left(D_{*}, A_{*}, Z\right) & =W-\tilde{W}+\tilde{C}_{*}^{\ll 23}-C_{*}^{\ll 23}, \\
G\left(\tilde{C}_{*}, D_{*}, A_{*}\right)-G\left(C_{*}, D_{*}, A_{*}\right) & =Z-\tilde{Z}+\tilde{B}_{*}^{\ll 19}-B_{*}^{\ll 19}-1,
\end{aligned}
$$

where $\tilde{B}_{*}$ stands for $B_{*}-1 \ll 25$ and $\tilde{C}_{*}$ stands for $C_{*}+1 \ll 5$. Equations (1) - (8) simply follow by elimination of $X_{\varphi(i)}$ from the two equations defining the steps $i=12, \ldots, 19$ of compress 12 for the inputs $X$ and $\tilde{X}$. As an example, by the definition of step 15 we have

$$
\begin{aligned}
& Z=\left(B+F(W, V, U)+X_{15}\right)^{\ll 19}, \\
& \tilde{Z}=\left(B+F(\tilde{W}, \tilde{V}, \tilde{U})+X_{15}\right)^{\ll 19}
\end{aligned}
$$

implying (4). Conversely, if a collection of solutions of (1) - (8) is given then we obtain an inner almost-collision by setting

$$
X_{13}=\text { arbitrary, }
$$




$$
\begin{aligned}
X_{14} & =W^{\ll 21}-C-F(V, U, B), \\
X_{15} & =Z^{\ll 13}-B-F(W, V, U), \\
X_{0} & =A_{*}^{\ll 29}-U-G(Z, W, V)-K_{1}, \\
X_{4} & =D_{*}^{\ll 27}-V-G\left(A_{*}, Z, W\right)-K_{1}, \\
X_{8} & =C_{*}^{\ll 23}-W-G\left(D_{*}, A_{*}, Z\right)-K_{1}, \\
X_{12} & =B_{*}^{\ll 19}-Z-G\left(C_{*}, D_{*}, A_{*}\right)-K_{1}, \\
D & =V^{\ll 25}-F(U, B, C)-X_{13}, \\
A & =U^{\ll 19}-F(B, C, D)-X_{12} .
\end{aligned}
$$

The system (1) - (8) has 14 variables. Thus it is a natural idea to make settings for some of the variables such that finding a solution for the remaining variables is feasible. Therefore we set

$$
U=-1=0 x f f f f f f f, \quad \tilde{U}=0, \quad B=0 .
$$

Then (1) is satisfied and (2), (3), (6), (7), (8) can be transformed and reordered as follows:

$$
\begin{aligned}
\tilde{Z} & =Z-G\left(\tilde{C}_{*}, D_{*}, A_{*}\right)+G\left(C_{*}, D_{*}, A_{*}\right)+\tilde{B}_{*}^{\ll 19}-B_{*}^{\ll 19}-1, \\
\tilde{W} & =W-G\left(D_{*}, A_{*}, \tilde{Z}\right)+G\left(D_{*}, A_{*}, Z\right)+\tilde{C}_{*}^{\ll 23}-C_{*}^{\ll 23}, \\
V & =W^{\ll 21}-\tilde{W}^{\ll 21}, \\
\tilde{V} & =V-G\left(A_{*}, \tilde{Z}, \tilde{W}\right)+G\left(A_{*}, Z, W\right), \\
C & =V^{\ll 25}-\tilde{V}^{\ll 25},
\end{aligned}
$$

For this system the variables $A_{*}, B_{*}, C_{*}, D_{*}, Z$ and $W$ form free parameters for the set of all solutions. The two remaining equations (4) and (5) are now

$$
\begin{aligned}
G(Z, W, V)-G(\tilde{Z}, \tilde{W}, \tilde{V}) & =1, \\
F(\tilde{W}, \tilde{V},-1)-F(W, V, 0)-\tilde{Z}^{\ll 13}+Z^{\ll 13} & =0 .
\end{aligned}
$$

\section{Algorithm searching for inner almost-collisions}

After this preparations we can give an example of an algorithm leading to solutions of (1) - (8), that is to an inner almost-collision, in about one second on a PC. The basic idea can be described as a kind of "continuous approximation" (cf. [4], Section 4).

1. Choose $A_{*}, B_{*}, C_{*}, D_{*}, Z, W$ randomly, compute $\tilde{Z}, \tilde{W}, V, \tilde{V}$ according to (18) - (21) and test (23). If the test is passed goto 2. (Since $W$ and $\tilde{W}$ (resp. $Z$ and $\tilde{Z}$ ) are close with respect to Hamming distance, there is a relatively high probability that (23) is satisfied.)

2. Take $A_{*}, B_{*}, C_{*}, D_{*}, Z, W$ found in 1. as "basic values". Change one random bit in each of these variables, compute the associated $\tilde{Z}, \tilde{W}, V, \tilde{V}$ and test if equation (23) is still satisfied and if, moreover, the left 4 bits of

$$
F(\tilde{W}, \tilde{V},-1)-F(W, V, 0)-\tilde{Z}^{\ll 13}+Z^{\ll 13}
$$


are zero. If this test is passed take the corresponding values $A_{*}, B_{*}, C_{*}, D_{*}$, $Z, W$ as the new "basic values". The next is doing the same as before, but now testing if the 8 left bits of (25), instead of 4, are zero. Continue with the 12, 16, ... left bits until (24) is fulfilled.

3. Now (23) and (24) are satisfied, and we obtain an inner almost-collision by setting $B=0$ and defining $A, C, D$, and $X_{i}(i=0,4,8,12,13,14,15)$ according to (9) - (17) and (22).

In order that the inner almost-collision can be used for the differential attack explained in the next section, the following additional equation has to be satisfied:

$$
G\left(B_{*}, C_{*}, D_{*}\right)=G\left(\tilde{B}_{*}, \tilde{C}_{*}, D_{*}\right) .
$$

Since $\tilde{B}_{*}$ and $B_{*}$ (resp. $\tilde{C}_{*}$ and $C_{*}$ ) are close, there is a high probability that this condition is true. Thus, to achieve also (26), the above step 2 . has to be repeated a few times. (To be more precise, 9 times on average, as we shall see in the next section.)

We call an inner almost-collision admissible if (26) is satisfied. Using again our original notation in (26) we can summarize the result of this section as follows:

Lemma 1. There is a practical algorithm, which allows to compute an admissible inner almost-collision, i.e. an initial value $(A, B, C, D)$ and inputs $X_{12}, X_{13}$, $X_{14}, X_{15}, X_{0}, X_{4}, X_{8}$ for compress 12 such that we have

$$
\begin{aligned}
\Delta_{19} & =\left(0,1^{\ll 25},-1^{\ll 5}, 0\right), \\
G\left(B_{19}, C_{19}, D_{19}\right) & =G\left(\tilde{B}_{19}, \tilde{C}_{19}, D_{19}\right) .
\end{aligned}
$$

The computation requires less than one second on a PC.

\section{Differential Attack Modulo $2^{32}$ (Step 20 to Step 35)}

The main part of the work has been done in the preceding section. We are now well-prepared for a routine differential attack, which will allow us to find collisions for the compress function of MD4. Using the notation introduced in Section 2 we can state this result as follows:

Lemma 2. Suppose that an admissible inner almost-collision, i.e. an initial value $(A, B, C, D)$ for step 12 and variables $X_{12}, X_{13}, X_{14}, X_{15}, X_{0}, X_{4}, X_{8}$ are given according to Lemma 1 . Choose the remaining $X_{i}$ 's randomly and determine the corresponding initial value by computing compress ${ }_{11}^{0}$ backwards starting with

$$
\left(A_{11}, B_{11}, C_{11}, D_{11}\right)=(A, B, C, D) .
$$

Then the probability that $X$ and $\tilde{X}$ form a collision for the compress function of $M D_{4}$ (i.e. $\Delta_{35}=0$ ) is about $2^{-22}$. 
Proof. Let $p$ be the probability that $\Delta_{\mathbf{3 5}}=0$ under the given assumption. We have to confirm that

$$
p \approx 2^{-22}
$$

The below table defines a sequence of fixed values $\Delta_{i}^{*}(i=19, \ldots, 35)$ for differences starting with $\Delta_{19}^{*}=\left(0,1^{\ll 25},-1 \ll 5,0\right)$ and ending with $\Delta_{35}^{*}=0$. The framed entries correspond to those variables which are modified in the particular steps. The Boolean functions $G$ and $H$ are majority and XOR, respectively.

\begin{tabular}{|c|c|c|c|c|c|c|c|c|c|}
\hline step $i$ & \multicolumn{4}{|c|}{$\Delta_{i}^{*}$} & fct & shift & $p_{i}^{i-1}$ & input & const \\
\hline 19 & 0 & $1 \ll 25$ & $-1 \ll 5$ & 0 & * & $*$ & $*$ & $*$ & $*$ \\
\hline 20 & 0 & $1 \ll 25$ & $-1 \ll 5$ & 0 & $G$ & 3 & 1 & $X_{1}$ & $K_{1}$ \\
\hline 21 & 0 & $1 \ll 25$ & $-1 \ll 5$ & 0 & $G$ & 5 & $1 / 9$ & $X_{5}$ & $K_{1}$ \\
\hline 22 & 0 & $1 \ll 25$ & $-1 \ll 14$ & 0 & $G$ & 9 & $1 / 3$ & $X_{9}$ & $K_{1}$ \\
\hline 23 & 0 & $1 \ll \theta$ & $-1 \ll 14$ & 0 & $G$ & 13 & $1 / 3$ & $X_{13}$ & $K_{1}$ \\
\hline 24 & 0 & $1 \ll B$ & $-1 \ll 14$ & 0 & $G$ & 3 & $1 / 9$ & $X_{2}$ & $K_{1}$ \\
\hline 25 & 0 & $1 \ll 6$ & $-1^{<14}$ & 0 & $G$ & 5 & $1 / 9$ & $X_{6}$ & $K_{1}$ \\
\hline 26 & 0 & $1 \ll 6$ & $-1 \ll 23$ & 0 & $G$ & 9 & $1 / 3$ & $X_{10}$ & $K_{1}$ \\
\hline 27 & 0 & $1 \ll 19$ & $-1 \ll 23$ & 0 & $G$ & 13 & $1 / 3$ & $X_{14}$ & $K_{1}$ \\
\hline 28 & 0 & $1 \ll 1 \theta$ & $-1 \ll 23$ & 0 & $G$ & 3 & $1 / 9$ & $X_{3}$ & $K_{1}$ \\
\hline 29 & 0 & $1 \ll 19$ & $-1 \ll 23$ & 0 & $G$ & 5 & $1 / 9$ & $X_{7}$ & $K_{1}$ \\
\hline 30 & 0 & $1 \ll 19$ & -1 & 0 & $G$ & 9 & $1 / 3$ & $X_{11}$ & $K_{1}$ \\
\hline 31 & 0 & 1 & -1 & 0 & $G$ & 13 & $1 / 3$ & $X_{15}$ & $K_{1}$ \\
\hline 32 & 0 & 1 & -1 & 0 & $H$ & 3 & $1 / 3$ & $X_{0}$ & $K_{2}$ \\
\hline 33 & 0 & 1 & -1 & 0 & $H$ & 9 & $1 / 3$ & $X_{8}$ & $K_{2}$ \\
\hline 34 & 0 & 1 & 0 & 0 & $H$ & 11 & $1 / 3$ & $X_{4}$ & $K_{2}$ \\
\hline 35 & 0 & 0 & 0 & 0 & $H$ & 15 & 1 & $X_{12}(+1)$ & $K_{2}$ \\
\hline
\end{tabular}

Here $p_{i}^{j}(i>j)$ denotes the probability that $\Delta_{i}=\Delta_{i}^{*}$ under the assumption that $\Delta_{j}=\Delta_{j}^{*}$. The asterisk entries for step 19 mean that we do not refer to these values in our argumentation. Note that $\Delta_{19}=\Delta_{19}^{*}$, since an inner almost-collision is given. We have $\Delta_{20}=\Delta_{20}^{*}$ and therefore $p_{20}^{19}=1$, because the given inner almost-collision is admissible. To verify $p_{\mathbf{3 5}}^{\mathbf{3 4}}=1$ note that $\Delta_{\mathbf{3 4}}=(0,1,0,0)=\Delta_{\mathbf{3 4}}^{*}$ implies

$$
\begin{aligned}
B_{35} & =\left(B_{34}+H\left(C_{34}, D_{34}, A_{34}\right)+X_{12}+K_{2}\right) \ll 15 \\
& =\left(\left(\tilde{B}_{34}+1\right)+H\left(\tilde{C}_{34}, \tilde{D}_{34}, \tilde{A}_{34}\right)+X_{12}+K_{2}\right)^{\ll 15} \\
& =\left(\tilde{B}_{34}+H\left(\tilde{C}_{34}, \tilde{D}_{34}, \tilde{A}_{34}\right)+\left(X_{12}+1+K_{2}\right)\right)^{\ll 15} \\
& =\tilde{B}_{35} .
\end{aligned}
$$


Also each other of the given values for $p_{i}^{i-1}$ can be proved easily. As an example, for $i=32$ we have to show that $(R+1) \oplus S=R \oplus(S+1)$ holds with probability $1 / 3$ for independent random words $R, S$. This equation is satisfied if and only if exactly one of the following conditions for the binary representations of $R$ and $S$ is given:

$$
\begin{aligned}
R & =* 0 \text { and } S=* 0, \\
R & =* 01 \text { and } S=* 01, \\
R & =* 011 \text { and } S=* 011, \\
& \ldots \\
& \ldots \\
& \ldots \\
R & =01 \ldots 11 \text { and } S=01 \ldots 11 \\
R & =1 \ldots 11 \text { and } S=1 \ldots 11 .
\end{aligned}
$$

Here an asterisk marks an arbitrary bit sequence of suitable length. (These sequences do not have to coincide for $R$ and $S$ in the particular cases.) Thus we conclude

$$
p_{32}^{31}=\frac{1}{2^{2}}+\frac{1}{4^{2}}+\frac{1}{8^{2}}+\cdots+\frac{1}{2^{62}}+\frac{1}{2^{64}}+\frac{1}{2^{64}}=\frac{1}{3}\left(1+\frac{1}{2^{63}}\right) .
$$

\begin{tabular}{|c|c|c|c|c|c|}
\hline step $i$ & \multicolumn{4}{|c|}{$\Delta_{i}^{*}$} & $p_{i}^{i-4}$ \\
\hline 19 & 0 & $1 \ll 25$ & $-1 \ll 5$ & 0 & * \\
\hline 23 & 0 & $1 \ll \theta$ & $-1 \ll 14$ & 0 & $1 / 35$ \\
\hline 27 & 0 & $1 \ll 18$ & $-1 \ll 23$ & 0 & $1 / 315$ \\
\hline 31 & 0 & 1 & -1 & 0 & $1 / 315$ \\
\hline 35 & 0 & 0 & 0 & 0 & $1 / 7$ \\
\hline
\end{tabular}

The above table yields

$$
\prod_{i=20}^{35} p_{i}^{i-1}=2^{-30.11}
$$

This already indicates that $p$ is large enough for a practical attack. Since the conditions $\Delta_{i}=\Delta_{i}^{*}$ are strongly dependent, we obtain a much more realistic approximation for $p$ if we consider four steps at once. The values for $p_{i}^{i-4}$ can certainly be computed similar as $p_{i}^{i-1}$. However, this seems to require lengthy considerations of various cases. The following values have been found by a simple Monte Carlo method.

Now we get

$$
\prod_{i=23}^{35} p_{i}^{i-4}=2^{-24.54}
$$


This is a much better approximation for $p$. Experimental observations suggest that $p$ is in fact still larger. We found the estimation $p \approx 2^{-22}$.

Thus we have shown that a random choice of the nine free $X_{i}$ 's gives a collision of the compress function with probability $2^{-22}$. Therefore, in principle, each given inner almost-collision allows to find on average about $2^{266}$ collisions for the compress functions.

Actually we need much less than $2^{22}$ trials to find a collision for the compress function. The reason is that we do not have to start each trial from the beginning. If $X_{1}, X_{5}, X_{9}$ have been found such that together with the already fixed $X_{13}$ we have reached the required difference for step 23, then keep $X_{1}, X_{5}, X_{9}$. Next choose suitable values for $X_{2}, X_{6}, X_{10}$, and so on. In this way we can find a collision for the compress function in a small fraction of a second on a PC.

\section{$5 \quad$ Right Initial Value (Step 0 to Step 11)}

It remains to compute collisions with the initial value $I V_{0}$ required by the definition of MD4. By Lemma 2 there are enough variables free to manage this easily. (The following argumentation does of course not depend on the particular choice of $I V_{0}$.)

Suppose an admissible inner almost-collision with initial value $(A, B, C, D)$ is given. Take random $X_{1}, X_{2}, X_{3}$ and $X_{5}$. Recall that $X_{0}, X_{4}$ and $X_{8}$ are already fixed. Compute compress ${ }_{5}^{0}\left(I V_{0} ; X_{0}, \ldots, X_{5}\right)$. Now $A_{5}=A_{4}, B_{5}=B_{4}=B_{3}$, $C_{5}=C_{4}=C_{3}=C_{2}, D_{5}$ are fixed.

Next we shall define $X_{6}, X_{7}, X_{9}, X_{10}$ and $X_{11}$ such that the output of compress $_{11}^{0}\left(I V_{0} ; X_{0}, \ldots, X_{11}\right)$ matches with $(A, B, C, D)$, or in other words

$$
\text { compress }_{11}^{6}\left(\left(A_{4}, B_{3}, C_{2}, D_{5}\right) ; X_{6}, \ldots, X_{11}\right)=(A, B, C, D) \text {. }
$$

Matching $B, C$ and $D$ can be done directly by associating suitable values to the free variables $X_{11}, X_{10}$ and $X_{9}$, respectively. It remains to match $A$ in step 8 . This cannot be done as before, since $X_{8}$ is already fixed. Step 8 is defined by the equation

$$
A_{8}=\left(A_{4}+F\left(B_{7}, C_{6}, D_{5}\right)+X_{8}\right)^{\ll 3} .
$$

According to the definition of $F$ as selection function we achieve $A_{8}=A$ if $B_{7}=-1=0 x f f f f f f f f$ and $C_{6}=A^{\ll 29}-A_{4}-X_{8}$. These values for $C_{6}$ and $B_{7}$ can be obtained by a suitable choice of $X_{6}$ and $X_{7}$.

Explicitly, this simple idea leads to the settings:

$$
\begin{aligned}
& X_{6}:=-C_{2}-F\left(D_{5}, A_{4}, B_{3}\right)+\left(A^{\ll 29}-A_{4}-X_{8}\right)^{\ll 21}, \\
& C_{6}=\left(C_{2}+F\left(D_{5}, A_{4}, B_{3}\right)+X_{6}\right)^{\ll 11}=A^{\ll 29}-A_{4}-X_{8}, \\
& X_{7}:=-B_{3}-F\left(C_{6}, D_{5}, A_{4}\right)-1, \\
& B_{7}=\left(B_{3}+F\left(C_{6}, D_{5}, A_{4}\right)+X_{7}\right)^{\ll 19}=-1, \\
& A_{8}=\left(A_{4}+F\left(-1, C_{6}, D_{5}\right)+X_{8}\right)^{\ll 3}=\left(A_{4}+C_{6}+X_{8}\right)^{\ll 3}=A,
\end{aligned}
$$




$$
\begin{aligned}
X_{9} & :=D^{\ll 25}-D_{5}-F\left(A,-1, C_{6}\right), \\
D_{9} & =\left(D_{5}+F\left(A,-1, C_{6}\right)+X_{9}\right)^{\ll 7}=D, \\
X_{10} & :=C^{\ll 21}-C_{6}-F(D, A,-1), \\
C_{10} & =\left(C_{6}+F(D, A,-1)+X_{10}\right)^{\ll 11}=C, \\
X_{11} & :=B^{\ll 13}+1-F(C, D, A), \\
B_{11} & =\left(-1+F(C, D, A)+X_{11}\right)^{\ll 19}=B .
\end{aligned}
$$

This means we obtain

$$
\begin{aligned}
\operatorname{compress}_{11}^{0}\left(I V_{0} ; X_{0}, \ldots, X_{11}\right) & =\left(A_{11}, B_{11}, C_{11}, D_{11}\right)=\left(A_{8}, B_{11}, C_{10}, D_{9}\right) \\
& =(A, B, C, D)
\end{aligned}
$$

i.e. as desired, we have reached the connection to the given inner almost-collision.

\section{Collision Search Algorithm}

As we now have described all parts of the attack, we give an overview summarizing the single steps of the derived algorithm searching for collisions:

1. Compute $A, B, C, D$ and $X_{0}, X_{4}, X_{8}, X_{12}, X_{13}, X_{14}, X_{15}$, which give an inner almost-collision (from step 12 to 19). The technical details of a suitable algorithm have been explained in Section 3. It also fixes values for $A_{19}, B_{19}, C_{19}, D_{19}$ and $\tilde{A}_{19}, \tilde{B}_{19}, \tilde{C}_{19}, \tilde{D}_{19}$.

2. According to Section 4 and 5 choose $X_{1}, X_{2}, X_{3}, X_{5}$ randomly and compute

$$
\begin{aligned}
\left(A_{5}, B_{5}, C_{5}, D_{5}\right) & =\text { compress }_{5}^{0}\left(I V_{0} ; X_{0}, \ldots, X_{5}\right), \\
t & =A^{\ll 29}-A_{5}-X_{8}, \\
X_{6} & =t^{\ll 21}-C_{5}-F\left(D_{5}, A_{5}, B_{5}\right), \\
X_{7} & =-1-B_{5}-F\left(t, D_{5}, A_{5}\right), \\
X_{9} & =D^{\ll 25}-D_{5}-F(A,-1, t), \\
X_{10} & =C^{\ll 21}-t-F(D, A,-1), \\
X_{11} & =B^{\ll 13}+1-F(C, D, A), \\
\left(A_{35}, B_{35}, C_{35}, D_{35}\right) & =\text { compress }_{35}^{20}\left(A_{19}, B_{19}, C_{19}, D_{19} ; X\right), \\
\left(\tilde{A}_{35}, \tilde{B}_{35}, \tilde{C}_{35}, \tilde{D}_{35}\right) & =\operatorname{compress}_{35}^{20}\left(\tilde{A}_{19}, \tilde{B}_{19}, \tilde{C}_{19}, \tilde{D}_{19} ; \tilde{X}\right), \\
\Delta_{35} & =\left(A_{35}, B_{35}, C_{35}, D_{35}\right)-\left(\tilde{A}_{35}, \tilde{B}_{35}, \tilde{C}_{35}, \tilde{D}_{35}\right) .
\end{aligned}
$$

3. If $\Delta_{35}=0$ then we have found a collision. Otherwise make a new trial by going to 2 . 


\section{Tuning, computational effort, and example}

To make 2. more effective do not compute the compress function from step 20 to step 35 completely. Instead, as condition to break up the trial, test immediately after each step $i$ if $\Delta_{i} \neq \Delta_{i}^{*}(i=21,22, \ldots)$; see first table in Section 4.

In this way for the largest part of all trials the computation will be broken up already after step 21, i.e. it is restricted to (27) - (33) and two steps of (34) and (35), respectively. (On the other hand it is unlikely that $\Delta_{35}=0$ but $\Delta_{i} \neq \Delta_{i}^{*}$ for some $i$. The sequence $\Delta_{19}^{*}, \ldots, \Delta_{34}^{*}$ in Section 3 is the "almost unique" way leading to $\Delta_{35}=0$. If we leave this way then it is very likely that the avalanche effect brings everything out of control. Hence it is unlikely that we loose a successful trial by the proposed selection.) Thus mostly a trial requires about the same effort as 16 steps (one third) of MD4-compress.

In view of Lemma 2 this means that the finding of a collision takes on average the same computational effort as about $2^{20}$ computations of MD4-compress. This estimation has been confirmed by an implementation of the attack.

The algorithm sometimes runs into a dead end. For instance, this happens if the values $A_{19}, B_{19}, C_{19}, D_{19}$ and $\tilde{A}_{19}, \tilde{B}_{19}, \tilde{C}_{19}, \tilde{D}_{19}$ coming from the inner almost-collision are bad-conditioned with respect to the differential attack. This effect can be taken into account by controlling the success of the algorithm and making a new start if necessary.

Beside the complexity of a collision search algorithm, the "variety" of collisions which, at least theoretically, can be found is another important aspect. In particular the number of obtainable collisions is of interest. We therefore mention that by Lemma 2 for each found inner almost-collision, in principle, about $2^{106}$ collisions of MD4 can be computed applying the above algorithm, since there are four words free in 2. (i.e. $2^{128}$ trials), and the probability for a success is about $2^{-22}$.

For the sake of readability of our exposition we have restricted ourselves to the description of the most direct version of our attack. But there are still many further technical tricks to improve it considerably. In this way we can even get collisions of practical relevance. This is demonstrated in the next section. First, however, we give a collsion which has been found by the previously described search algorithm:

$$
\begin{array}{llll}
X_{0}=0 x 13985 e 12 & X_{4}=0 x 2 d 6 e 09 a c & X_{8}=0 x a b e 17 b e 0 & X_{12}=0 x 20771027 \\
X_{1}=0 x 748 a 810 b & X_{5}=0 x 4 b 6 d b d b 9 & X_{9}=0 x e d 1 e d 4 b 3 & X_{13}=0 x \text { fdfffbff } \\
X_{2}=0 x 4 d 1 d f 15 a & X_{6}=0 x 6464 b 0 c 8 & X_{10}=0 x 4120 a b f 5 & X_{14}=0 x f f f f b f f b \\
X_{3}=0 x 181 d 1516 & X_{7}=0 x f b a 1 c 097 & X_{11}=0 x 20771029 & X_{15}=0 x 6774 b e d 2
\end{array}
$$

Recall that $\tilde{X}$ is defined by setting $\tilde{X}_{i}=X_{i}(i<16, i \neq 12)$ and

$$
\tilde{X}_{12}=X_{12}+1=0 x 20771028 \text {. }
$$

$X$ and $\tilde{X}$ have the same MD4-compress value with respect to the initial value $I V_{0}$ (see Appendix). The complete MD4 algorithm, including the padding rule, associates to $X$ and $\tilde{X}$ the common hash value 


\section{Collisions for Crooks}

How to swindle Ann (cf. [10])

Alf wanted to sell Ann his house, and Ann was interested. They agreed on a price of $\$ 176,495$. Alf asked Ann to sign a contract using a digital signature scheme which is based on some public-key algorithm and the hash function MD4. The contract read as follows:

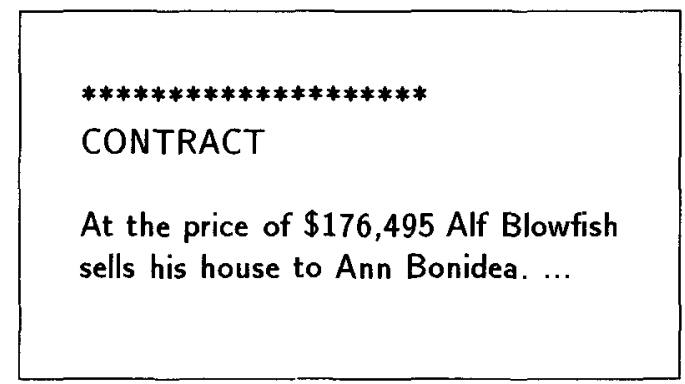

"The first 20 bytes (each of them is represented by an asterisk above) are random. They have been placed before the text for security reasons!" claimed Alf, and Ann signed the contract. Later, however, Alf substituted the contract file by another which read as follows:

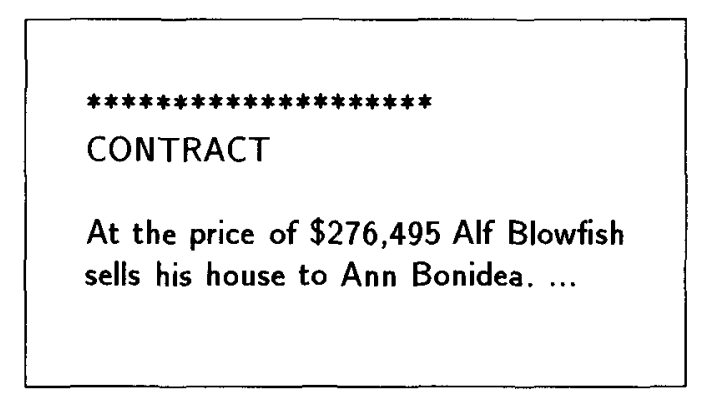

The contract had been prepared by him such that replacing $\$ 176,495$ by $\$ 276,495$ does not change the MD4 hash value!

\section{How Alf did it}

We shall now explain the precise definition of the above digital contract. Its first sixteen 32-bit words are:

$$
\begin{array}{lllll}
M_{0}=0 x 9074449 b & M_{4}=0 x 63247 e 24 & M_{8}=0 x 68742074 & M_{12}=0 x 2 C 363731 \\
M_{1}=0 x 1089 f c 26 & M_{5}=0 x 4 e 4 f 430 a & M_{9}=0 x 72702065 & M_{13}=0 x 20353934 \\
M_{2}=0 x 8 b f 37 f a 2 & M_{6}=0 x 43415254 & M_{10}=0 x 20656369 & M_{14}=0 x 20666 c 41 \\
M_{3}=0 x 1 d 630 d a f & M_{7}=0 x 410 a 0 a 54 & M_{11}=0 x 2420666 f & M_{15}=0 x 776 f 6 C 42
\end{array}
$$


The twenty bytes of $M_{0}-M_{4}$ are the above mentioned "random bytes". The bytes of $M_{5}$, in reverse ordering (according to the definition of MD4) and interpreted as ASCII read as follows:

$$
0 \text { a } 43 \text { 4f } 4 \mathbf{e}=\text { Line feed 'CON', }
$$

and so on to $M_{15}$ which reads

$$
426 \mathrm{c} \text { 6f } 77=\text { 'Blow'. }
$$

The sequence $M_{i}(i<16)$ has been chosen such that setting $M_{12}^{\prime}=M_{12}+1$ and $M_{i}^{\prime}=M_{i}$ for $i<16, i \neq 12$ gives a collision, i.e.

$$
\operatorname{compress}\left(I V_{0} ; M\right)=\operatorname{compress}\left(I V_{0} ; M^{\prime}\right)
$$

for the compress function of MD4 and its fixed initial value $I V_{0}$. This collision has been found in less than one hour on a PC. Interpreting $M_{12}=0 x 2 c 363731$ and $M_{12}^{\prime}=0 x 2 c 363732$ we get:

$$
\begin{aligned}
& M_{12}=3137362 \mathrm{c}=\text { ' } 176 \text {,' } \\
& M_{12}^{\prime}=3237362 \mathrm{c}=\text { '276,' }
\end{aligned}
$$

In view of the definition of MD4 as the iterative application of compress we obtain a collision by taking any bit string and appending it to $M$ and $M^{\prime}$.

\section{Conclusions}

A dedicated hash function should be secure and fast at the same time. Everyone who comes up with a new design of a fast algorithm, especially if there is not already sufficient experience with related algorithms, runs a great risk to overlook weaknesses and to underestimate possibilities of finding new cryptanalytic methods. But there is no other way than to start with concrete proposals and thereby pushing on an evolutionary process leading to better and better solutions. Therefore the introducing of MD4 by Ron Rivest [7] in 1990 was a significant contribution. Today there is a whole family of hash functions based on MD4's design elements.

A short time after MD4 had been introduced, some weaknesses became apparent and Rivest introduced MD5 in 1991. He explained his reasons in [8]:

"The MD5 algorithm is an extension of the MD4 message-digest algorithm. MD5 is slightly slower than MD4, but is more "conservative" in design. MD5 was designed because it was felt that MD4 was perhaps being adopted for use more quickly than justified by the existing critical review; because MD4 was designed to be exceptionally fast, it is "at the edge" in terms of risking successful cryptanalytic attack. ..." 
The weaknesses of MD4 observed in [3] and [9], two-round attacks and almost collisions, were generally considered to be mainly of theoretical importance. Now in view of the presented attack this can no longer be assumed, as has been demonstrated.

Where MD4 is still in use, it should be replaced!

An exception is the application of MD4 as a one-way function. What are possible alternatives? - The compress function of the 256-bit extension of MD4 (see [7]) is not collision-free [5]. RIPEMD is another strengthened mode of MD4 proposed in 1992 [2]. The design of RIPEMD and that of extended MD4 are very similar. We anticipate that, in addition to the already known two-round attacks [4], the compress function of RIPEMD is also not collision-free.

We have some reservations about MD5 as well. Although we think that MD5 is much stronger than MD4 and stronger than extended MD4, it might still turn out that the adding of one round and the other changes are not sufficient to protect MD5 against the methods developed in [4] and the present note.

As replacements for MD4 and MD5 we would suggest RIPEMD-160 (with 160-bit hash values) or RIPEMD-128 (with 128-bit hash values). These are described in [6] and have been designed as a strengthened version of RIPEMD, taking account of recent progress in the cryptanalysis of the MD4 family of hash functions.

Another alternative is the Secure Hash Algorithm (SHA-1), which was designed by NSA and published by NIST (National Institute of Standards and Technology, US) [1]. However, its design criteria are secret.

Acknowledgment. The author thanks Antoon Bosselaers for reading an earlier version of this paper very carefully and giving several hints improving the exposition.

\section{References}

1. FIPS 180-1, Secure hash standard, Federal Information Pracessing Standard, NIST, US Department of Commerce, Washington D.C., April 1995.

2. RIPE, Integrity Primitives for Secure Information Systems, Final Report of RACE Integrity Primitives Evaluation (RIPE-RACE 1040), Lecture Notes in Computer Science, vol. 1007, Springer-Verlag, 1995.

3. den Boer, B., Bosselaers, A.: An attack on the last two rounds of MD4, Advances in Cryptology, CRYPTO '91, Lecture Notes in Computer Science, vol. 576, SpringerVerlag, 1992, pp. $194-203$.

4. Dobbertin, H.: RIPEMD with two-round compress function is not collision-free, J. of Cryptology, to appear.

5. Dobbertin, H.: The compress function of extended MD4 is not collision-free, preprint.

6. Dobbertin, H., Bosselaers, A., Preneel, B.: RIPEMD-160: A strengthened version of RIPEMD, these proceedings. 
7. Rivest, R.: The MD4 message-digest algorithm, Request for Comments (RFC) 1320, Internet Activities Board, Internet Privacy Task Force, April 1992.

8. Rivest, R.: The MD5 message-digest algorithm, Request for Comments (RFC) 1321, Internet Activities Board, Internet Privacy Task Force, April 1992.

9. Vaudenay, S:: On the need of multipermutations: Cryptanalysis of MD4 and SAFER, Fast Software Encryption (Proceedings of the 1994 Leuven Workshop on Cryptographic Algorithms), Lecture Notes in Computer Science, vol. 1008, Springer-Verlag, 1995, pp. 286 - 297.

10. Yuval, G.: How to swindle Rabin, Cryptologia, vol. 3, no. 3, 1979, pp. 187-189.

\section{Appendix}

The hash function MD4 is defined as the iteration of a certain compress function, which we shall specify below. The computation starts with the initial value

$$
I V_{0}=0 x 674523010 x e f c d a b 890 x 98 b a d c f e \quad 0 x 10325476 .
$$

Each application of the compress function uses a collection of four words as initial value and 16 words of the message as input, and it gives four words output, which are then used as initial value for the next application. The final output is the hash value. This works, since there is a padding rule (addition of bits to the message such that its length is a multiple of $512=16 \times$ (length of words)). A description of MD4 including also the padding rule can be found in [7].

The compress function of MD4 uses the Boolean vector functions

$$
\begin{aligned}
& \left.F^{\prime} U, V, W\right)=(U \wedge V) \vee(\neg U \wedge W), \\
& G(U, V, W)=(U \wedge V) \vee(U \wedge W) \vee(V \wedge W), \\
& H(U, V, W)=U \oplus V \oplus W
\end{aligned}
$$

and the constants

$$
\begin{aligned}
& K_{1}=0 x 5 a 827999 \\
& K_{2}=0 x 6 e d 9 e b a 1
\end{aligned}
$$

Let $F F(a, b, c, d, Z, s), G G(a, b, c, d, Z, s)$ and $H H(a, b, c, d, Z, s)$ denote the operations

$$
\begin{aligned}
& a:=(a+F(b, c, d)+Z)^{\ll \bullet} \\
& a:=(a+G(b, c, d)+Z)^{\ll}, \\
& a:=(a+H(b, c, d)+Z)^{\ll},
\end{aligned}
$$

respectively. In order to define the MD4 compress function suppose now that the initial value $(A, B, C, D)$ and inputs $X_{0}, X_{1}, \ldots, X_{15}$ are given. Copy $A, B, C, D$ into registers $a, b, c, d$, and apply the following steps: 


\section{First round}

$\begin{array}{ll}\text { step 0 } & F F\left(a, b, c, d, X_{0}, 3\right) \\ \text { step 1 } & F F\left(d, a, b, c, X_{1}, 7\right) \\ \text { step 2 } & F F\left(c, d, a, b, X_{2}, 11\right) \\ \text { step 3 } & F F\left(b, c, d, a, X_{3}, 19\right) \\ \text { step 4 } & F F\left(a, b, c, d, X_{4}, 3\right) \\ \text { step 5 } & F F\left(d, a, b, c, X_{5}, 7\right) \\ \text { step 6 } & F F\left(c, d, a, b, X_{6}, 11\right) \\ \text { step 7 } & F F\left(b, c, d, a, X_{7}, 19\right) \\ \text { step 8 } & F F\left(a, b, c, d, X_{8}, 3\right) \\ \text { step 9 } & F F\left(d, a, b, c, X_{9}, 7\right) \\ \text { step 10 } & F F\left(c, d, a, b, X_{10}, 11\right) \\ \text { step 11 } & F F\left(b, c, d, a, X_{11}, 19\right) \\ \text { step 12 } & F F\left(a, b, c, d, X_{12}, 3\right) \\ \text { step 13 } & F F\left(d, a, b, c, X_{13}, 7\right) \\ \text { step 14 } & F F\left(c, d, a, b, X_{14}, 11\right) \\ \text { step 15 } & F F\left(b, c, d, a, X_{15}, 19\right)\end{array}$

\section{Second round}

step $16 G G\left(a, b, c, d, X_{0}+K_{1}, 3\right)$

step $17 G G\left(d, a, b, c, X_{4}+K_{1}, 5\right)$

step $18 G G\left(c, d, a, b, X_{8}+K_{1}, 9\right)$

step $19 G G\left(b, c, d, a, X_{12}+K_{1}, 13\right)$

step $20 G G\left(a, b, c, d, X_{1}+K_{1}, 3\right)$

step $21 G G\left(d, a, b, c, X_{5}+K_{1}, 5\right)$

step $22 G G\left(c, d, a, b, X_{9}+K_{1}, 9\right)$

step $23 G G\left(b, c, d, a, X_{13}+K_{1}, 13\right)$

step $24 G G\left(a, b, c, d, X_{2}+K_{1}, 3\right)$

step $25 G G\left(d, a, b, c, X_{6}+K_{1}, 5\right)$

step $26 G G\left(c, d, a, b, X_{10}+K_{1}, 9\right)$

step $27 G G\left(b, c, d, a, X_{14}+K_{1}, 13\right)$

step $28 G G\left(a, b, c, d, X_{3}+K_{1}, 3\right)$

step $29 G G\left(d, a, b, c, X_{7}+K_{1}, 5\right)$

step $30 G G\left(c, d, a, b, X_{11}+K_{1}, 9\right)$

step $31 G G\left(b, c, d, a, X_{15}+K_{1}, 13\right)$

\section{Third round}

$$
\begin{array}{ll}
\text { step 32 } H H\left(a, b, c, d, X_{0}+K_{2}, 3\right) \\
\text { step 33 } H H\left(d, a, b, c, X_{8}+K_{2}, 9\right) \\
\text { step 34 } H H\left(c, d, a, b, X_{4}+K_{2}, 11\right) \\
\text { step 35 } H H\left(b, c, d, a, X_{12}+K_{2}, 15\right) \\
\text { step 36 } H H\left(a, b, c, d, X_{2}+K_{2}, 3\right) \\
\text { step 37 } H H\left(d, a, b, c, X_{10}+K_{2}, 9\right) \\
\text { step 38 } H H\left(c, d, a, b, X_{6}+K_{2}, 11\right) \\
\text { step 39 } H H\left(b, c, d, a, X_{14}+K_{2}, 15\right) \\
\text { step 40 } H H\left(a, b, c, d, X_{1}+K_{2}, 3\right) \\
\text { step 41 } H H\left(d, a, b, c, X_{8}+K_{2}, 9\right) \\
\text { step 42 } H H\left(c, d, a, b, X_{5}+K_{2}, 11\right) \\
\text { step 43 } H H\left(b, c, d, a, X_{13}+K_{2}, 15\right) \\
\text { step 44 } H H\left(a, b, c, d, X_{3}+K_{2}, 3\right) \\
\text { step 45 } H H\left(d, a, b, c, X_{11}+K_{2}, 9\right) \\
\text { step 46 } H H\left(c, d, a, b, X_{7}+K_{2}, 11\right) \\
\text { step 47 } H H\left(b, c, d, a, X_{15}+K_{2}, 15\right)
\end{array}
$$

Finally compute the output $A A, B B, C C, D D$ as follows:

$$
A A=A+a, \quad B B=B+b, \quad C C=C+c, \quad D D=D+d .
$$

That is one sets

$$
\text { compress }\left((A, B, C, D) ; X_{0}, X_{1}, \ldots, X_{15}\right)=(A A, B B, C C, D D) .
$$

